\title{
Molecular logic computing model based on self-assembly of DNA nanoparticles
}

\author{
ZHANG Cheng $^{1 *}$, YANG Jing ${ }^{1,2^{*}} \&$ XU Jin ${ }^{1 *}$ \\ ${ }^{1}$ Institute of Software, School of Electronics Engineering and Computer Science, Key Laboratory of High Confidence Software Technologies of \\ Ministry of Education, Peking University, Beijing 100871, China; \\ ${ }^{2}$ Institute of Computing Technology, Chinese Academy of Sciences, Beijing 100190, China
}

Received May 27, 2011; accepted August 4, 2011

\begin{abstract}
In this paper, a logic computing model was constructed using a DNA nanoparticle, combined with color change technology of DNA/Au nanoparticle conjugates, and DNA computing. Several important technologies are utilized in this molecular computing model: DNA self-assembly, DNA/Au nanoparticle conjugation, and the color change resulting from Au nanoparticle aggregation. The simple logic computing model was realized by a color change, resulting from changing of DNA self-assembly. Based on this computing model, a set of operations computing model was also established, by which a simple logic problem was solved. To enlarge the applications of this logic nanocomputing system, a molecular detection method was developed for H1N1 virus gene detection.
\end{abstract}

logic computing, DNA computing, nanoparticle, DNA self-assembly

Citation: Zhang C, Yang J, Xu J. Molecular logic computing model based on self-assembly of DNA nanoparticles. Chinese Sci Bull, 2011, 56: 3566-3571, doi: $10.1007 / \mathrm{s} 11434-011-4725-3$

In the area of computing research, molecular computing has gradually grown from pure theory to experimental realization. In particular, DNA computing has become a leader in molecular computing, because of its advantages in nanotechnology operations. At the beginning of DNA computing, scientists attempted to solve certain difficult mathematical problems, such as the satisfiability problem, the graph coloring problem, the maximal clique problem, and the maximum independent set problem [1-4]. Despite the difficulty in comparing DNA computing, with traditional electronic computing, its potential in huge parallel computing remains an attractive focus.

In the area of information science, logic computing is a basic unit of computing. Thus, it is important to utilize molecules to implement logic computing, in which the molecules play a significant role. Many kinds of materials could be used to construct to logic gates, such as nucleic

*Corresponding authors (email: zhang_cheng@pku.edu.cn; yangjing@ict.ac.cn; jxu@pku.edu.cn) acids, and enzymes [5-11]. Logic systems based on DNA have attracted the most attention [12-15]. Recently, the combination of DNA self-assembly and nanoparticles has become a research hot spot [16-19]. A nanoparticle is a type of tiny particle at the nanometers scale. Commonly used nanoparticles are $\mathrm{Au}, \mathrm{Ag}$, and semi-conductor quantum dots. Importantly, the most widely used is the gold nanoparticle (AuNP), which is stable, uniform, and has strong chemical binding ability.

In this paper, a logic computing model was constructed using DNA nanoparticles, combined with the color change technology of the DNA/Au nanoparticle conjugates and DNA computing. The simple logic computing is realized by a color change, resulting from a change in DNA self-assembly. Meanwhile, a set operation computing model was also established, by which a simple logic problem was solved. To enlarge the applications of this logic nanocomputing system, a molecular detection method was developed for H1N1 virus gene detection. Importantly, several critical technologies are utilized in this molecular computing model: DNA 
self-assembly, DNA/Au nanoparticle conjugation and color changing by Au nanoparticle aggregation.

\section{Background introduction}

\subsection{Logic computing based on DNA}

With the development of nanotechnology of electronics and molecular information processing, DNA logic computing has attracted more attention. Not only has it been applied to gene engineering and disease diagnosis, DNA logic computing also has applications in parallel computing, nanoinformation processing, and molecular cryptography. In 2000, Mao first reported a simple DNA logic machine using DNA self-assembly in the journal Nature [20]. In 2003, Stojanovic constructed DNA logic computing models using DNAase [21]. Later, in 2006, another DNA logic computing system was reported in Science by a research group from the California Institute of Technology [22]. In 2010, Itamar Willner's research group established the molecular computing models utilizing DNAzymes, and this work was assessed as an innovative and systematic research [23]. All these studies provide directions of future efforts. However, most of the research has used DNA or enzymes, and thus the unit structures are rather simple. In addition, the detection methods can be somewhat complicated. Indeed, traditional detection methods have certain drawbacks, such as a multiplicity of procedures, long-term costs and the reliability of large equipment [24-26]. Therefore, there remain certain aspects that can be improved in DNA logic computing models.

\subsection{Color changes during self-assembly of DNA/AuNP conjugates}

The precise control of the formation of DNA nanomolecules has always been a research goal. To this end, the method of color change by DNA/AuNP conjugation has developed rapidly. The principle is that a random nanoparticle system is precisely controlled by DNA/AuNP self-assembly. For AuNP (quantum size, nanosurface), as the distance between nanoparticles changes, the absorption of the whole system will change accordingly. Therefore, at the macroscopic level, the reaction system will change color (Figure 1), and tiny difference in molecules can be observed as obvious color changes. The characteristics of the color changing reaction are stable. In addition, the time and steps needed for detection are much fewer and, because the results can be detected by the naked eye, large equipment is unnecessary.

In 1996, Mirkin et al. [27] reported the first self-assembly DNA/AuNP color change system in Nature. Thereafter, more research was performed in the areas of multiple molecular signal detection, molecular computing, nanochip, nanoelectronics, and gene engineering. To date, the research has become multidisciplinary in focus, including molecular information processing, molecular computing, and nanotechnology.

\section{Experimental methods}

\subsection{Logic algorithm, self-assembly structure, and DNA sequence design}

In traditional electronic computers, data is processed by binary system electronic or optical information, in which " 0 " denotes a lower voltage and " 1 " denotes a higher voltage. Basic logic computing includes three logic systems: AND, OR, and YES. The YES gate is the simplest logic device. In the YES gate system, the result value depends on the following relationship: input 1 produces output 1 ; input 0 produces output 0 . In the OR logic system, the true result is produced only if either or both of two input values are 1 . In the AND logic system, the true result is produced only when both of the input values are 1 . It is impossible to fulfill all logic computing just using these three logic gates; therefore, more complicated logic gates have been established, such as XOR and XNOR gates. Besides, set operation can also be implemented by logic computing. The set means an assembly including something with the same characteristics. In the end of the 19th century, German mathematicians enlarged the concepts of the finite set into the infinite set. After that, the set operation became a basic

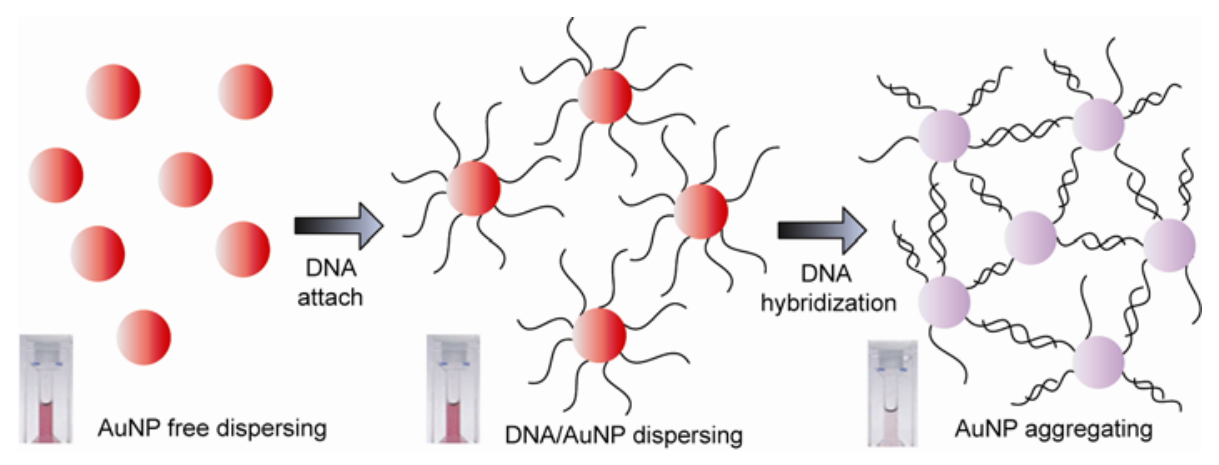

Figure 1 The principle of the aggregation of DNA/AuNP conjugates and the color change. 
branch in modern mathematics. The set operation plays an important role in the areas of computing, information, and cryptography.

In this experiment, the DNA self-assembly structures are used as the basic computing models. The results are obtained by DNA hybridization and DNA/AuNP color changes. Based on the logic computing models, the simple set operations are also carried out. The specific methods and steps are as follows:

Step 1: Construction of DNA/AuNP logic computing models

In the course of establishing logic computing models, there are two chief aspects: DNA sequence design and DNA/ AuNP self-assembly structure. First, the DNA/AuNP selfassembly structures are established according to the computing principle. Second, the appropriate DNA sequences are produced by various constraints. During DNA sequence design, hairpin structures, polymers, and mismatches should be avoided. To conveniently control hybridization, a length of $10 \mathrm{bp}$ is chosen for the hybridization sites. Then, the length of the informative DNA is $20 \mathrm{bp}$ (two sections, front and back). Additionally, the length of DNA strand that directly links to the AuNP is set at $15 \mathrm{bp}$, including the "arm region" consisting of a poly-T (5 bp) region. The DNA/ AuNP self-assembly structures used in this work are shown in Figure 2. One is a double-stranded DNA with no missing structures, which can self-assemble and connect the AuNP with each other, resulting in aggregation. The second structure has a missing of double-stranded DNA, and is not able to form self-assembly structures or cause aggregation. The DNA sequences used in this experiment are shown in Table 1.

Step 2: Add DNA strands containing different information. According to the sequences of the specific DNA strand, different hybridization structures and results are produced.

Step 3: By detecting aggregation of DNA/AuNP conjugates, the computing results are output from the logic computing models.

\subsection{Conjugation of DNA and AuNP}

The key method used in this work is conjugation of DNA and AuNP. $15 \mathrm{~nm}$ AuNP and thiol-DNA strands were used in the experiment. The specific steps were as follows:

(1) The thiol-DNA strands were activated by dithiothreitol (DTT) and then extracted twice by ethyl acetate.

(2) The "activated" thiol-DNA samples were added to the AuNP solution directly to the final concentration of $1 \mu \mathrm{M}$ $\left(1 \mathrm{M}=1 \mathrm{~mol} \mathrm{~L}^{-1}\right)$, and incubated for $24 \mathrm{~h}$.

(3) Then, an "Aging process" was carried out. Sodium polyphosphate buffer ( $\mathrm{pH} 7.0$ ) was added to $0.01 \mathrm{M}$. Later, $\mathrm{NaCl}$ was gradually added to a concentration to $0.2 \mathrm{M}$. The

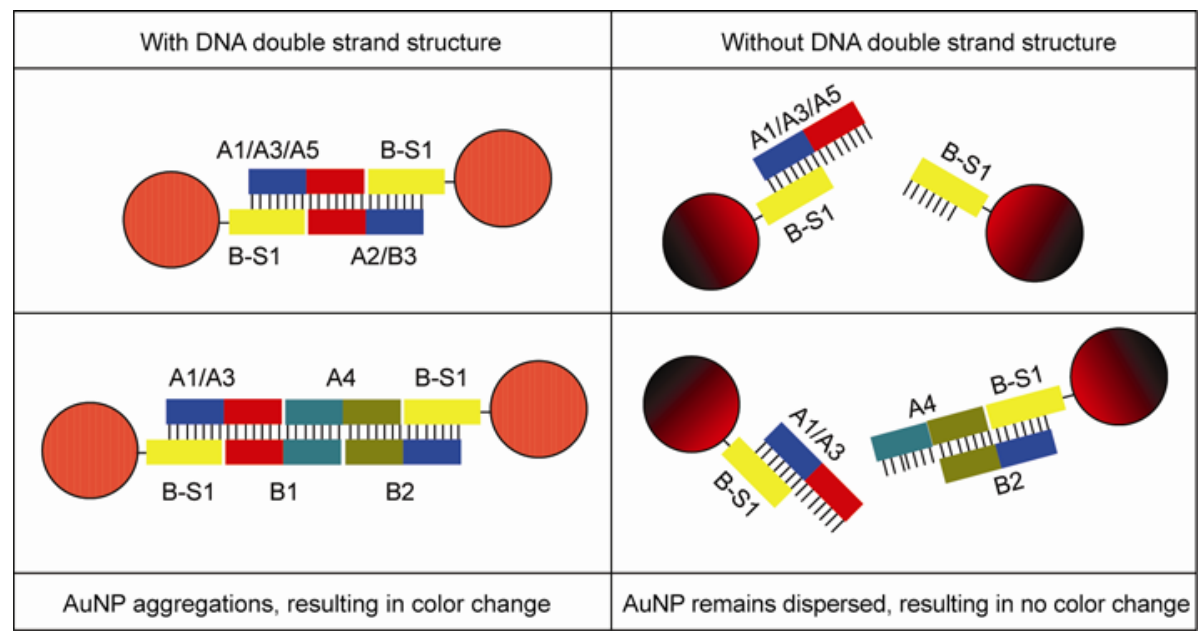

Figure 2 DNA structures and resultant color changes (names of DNA occupying the position are divided by “/”).

Table 1 DNA sequences used in this study ${ }^{\text {a) }}$

\begin{tabular}{|c|c|c|c|}
\hline Name & Sequence $\left(5^{\prime}\right.$ to $\left.3^{\prime}\right)$ & Name & Sequence $\left(5^{\prime}\right.$ to $\left.3^{\prime}\right)$ \\
\hline A1 & TCTGGCACTATGACAAGCGA & B-S1 & TAGTGCCAGATTTTT-Sh ${ }^{\mathrm{a})}$ \\
\hline A 2 & TCTGGCACTATCGCTTGTCA & B1 & CCCTGCTTCGTCGCTTGTCA \\
\hline A3 & TTCTGGCACTATTGACAAGCGA & $\mathrm{B} 2$ & TCTGGCACTACATACGCATT \\
\hline A4 & CGAAGCAGGGAATGCGTATG & B3 & TCTGGCACTACACAAGACAAGGTTT \\
\hline A5 & TCTGGCACTAAAACCTTGTCT & & \\
\hline
\end{tabular}


samples were then incubated for $40 \mathrm{~h}$.

(4) Unbound DNA was removed by centrifugation at $13200 \mathrm{r} \mathrm{min}^{-1}$ for $30 \mathrm{~min}$.

(5) The obtained DNA/AuNP conjugates were redissolved in sodium polyphosphate buffer ( $\mathrm{pH} 7.0)$, and stored at $4^{\circ} \mathrm{C}$.

The main materials are as follows: Disodium hydrogen phosphate, sodium dihydrogen phosphate, $\mathrm{NaCl}$, DTT, ethyl acetate (All chemicals were of analytical grade); $15 \mathrm{~nm}$ AuNP from Tedpella; All DNA samples were from Shanghai Sangon. The equipment: Alpha Imager, Anke TGL-16G centrifuge, Bio photometer (eppendorf), ELGA Ultra-Pure Water System, Sartorius electronic balance.

\subsection{Computing model reaction conditions}

In each reaction system, the volume of AuNP solution labeled by specific DNA strands was $100 \mu \mathrm{L}$. DNA samples were added to a concentration of $1 \mu \mathrm{M}$, and $\mathrm{NaCl}$ was added to $0.2 \mathrm{M}$. The samples were then incubated at $80^{\circ} \mathrm{C}$ for $10 \mathrm{~min}$ before being gradually cooled to room temperature. At the end of the reaction, the experimental results were recorded (color change or no color change). All reactions were performed in triplicate with independent samples.

\section{Experimental results}

\subsection{Computing results of basic logic models}

The logic computing models were constructed based on the DNA self-assembly structures in Figure 2. Where the DNA double strand structure was intact, the AuNPs were able to be connected by them, and AuNP aggregates were formed. This caused the absorption of the system to decrease, and the color to change from red to purple. In contrast, when the samples lacked certain components of the DNA double strand structures, AuNPs could not be connected, and no aggregation was produced. Under these conditions, the absorption of the system and the color showed no obvious changes (Figure 3).

The "YES" gate is the simplest logic device, in which there is only one information signal added. In the logic model, the result value depends on the following relationship: input 1 produces output 1 ; input 0 produces output 0 . In this model, the component includes: DNA/AuNP conjugates (attached with DNA strand B-S1), and DNA strand A1 (Figure 3). In the course of operation, DNA strand A2 was added as the information signal. When the signal was input (value 1 was added), the result was produced (color change). By contrast, without input signal (value 0 was added), no result was obtained (color did not change). From the view of DNA/AuNP self-assembly structures, when no signal was input, DNA strand A1 was only able to connect one end of AuNP, and produce no aggregation (Figure 2). To make detecting convenient, the control group was established with no DNA signals added.

In the "OR" logic system, the true result is produced only if either or both of two input values are 1. In the experiment, the model component includes: DNA/AuNP conjugates (attached with DNA strand B-S1), and DNA strand A2 (Figure 3). The information signal DNA strands were A1 and A3. Adding DNA strands A1 or A3 individually, the results were both 1 (color changing). When adding DNA strands $\mathrm{A} 1$ and $\mathrm{A} 3$ at the same time, the computing results were still 1 (color changing).

In the "AND" logic system, the true result is produced only when both of the input values are 1. "AND" logic model was operated in three ways. The model component includes: DNA/AuNP conjugates (attached with DNA strand B-S1, Figure 3). When signal DNA strands A1 or A2 was added individually, there was no color changing produced (results output 0). However, when signal DNA strands A1 and A2 was added at the same time, the model had color changing results (results output 1).

\subsection{Simple set operation results}

To test the potentiality and stability of the logic computing models, the set operation models were established (Figure 4). The problem example is as follows: let $(A \cup B) \cap(C \cap$ $\mathrm{D})=1$, answer $\mathrm{Xi}(i=1,2,3,4,5,6)$ in different conditions.
(1) $\mathrm{A}=\mathrm{X}_{1}, \mathrm{~B}=1, \mathrm{C}=1, \mathrm{D}=1$;
(2) $\mathrm{A}=1, \mathrm{~B}=\mathrm{X}_{2}, \mathrm{C}=1, \mathrm{D}=1$;
(3) $\mathrm{A}=1, \mathrm{~B}=0, \mathrm{C}=\mathrm{X}_{3}, \mathrm{D}=1$;

\begin{tabular}{l|c|c|c|}
\hline \multicolumn{4}{|c|}{ "YES" logic computing model } \\
\hline $\begin{array}{l}\text { Model } \\
\text { components }\end{array}$ & control & \multicolumn{2}{|c}{$\begin{array}{c}\text { DNA/AuNP } \\
\text { DNA: A1 }\end{array}$} \\
\hline $\begin{array}{l}\text { Input signal } \\
\text { DNA: A2 }\end{array}$ & 0 & 0 & 1 \\
\hline $\begin{array}{l}\text { Output } \\
\text { results }\end{array}$ & 0 & 0 & 1 \\
\hline
\end{tabular}

\begin{tabular}{|c|c|c|c|c|}
\hline \multicolumn{5}{|c|}{ "OR" logic computing model } \\
\hline \multirow{2}{*}{$\begin{array}{l}\text { Model } \\
\text { components }\end{array}$} & \multirow{3}{*}{\begin{tabular}{|c|} 
control \\
0
\end{tabular}} & \multicolumn{3}{|c|}{$\begin{array}{l}\text { DNA/AuNP } \\
\text { DNA: A2 }\end{array}$} \\
\hline & & & & \\
\hline DNA: A1 & & 1 & 0 & 1 \\
\hline DNA: A3 & 0 & 0 & 1 & 1 \\
\hline \multirow{2}{*}{$\begin{array}{l}\text { Output } \\
\text { results }\end{array}$} & 0 & 1 & 1 & 1 \\
\hline & t & f & e & $\theta$ \\
\hline
\end{tabular}

\begin{tabular}{l|c|c|c|c}
\multicolumn{4}{|c|}{ "AND" logic computing model } \\
\hline \begin{tabular}{l|c} 
Model \\
components
\end{tabular} & control & \multicolumn{4}{|c}{ DNAVAuNP } \\
\hline Input signal & & & & \\
DNA: A1 & 0 & 1 & 0 & 1 \\
DNA: A2 & 0 & 0 & 1 & 1 \\
\hline Output & 0 & 0 & 0 & 1 \\
results & $y$ & $y$ & $y$ & $y$ \\
\hline
\end{tabular}

Figure 3 Results of nanoparticle logic computing models. 


\begin{tabular}{|c|c|c|c|c|c|c|c|c|c|c|c|c|}
\hline \multirow{3}{*}{$\begin{array}{l}\text { Model } \\
\text { component }\end{array}$} & & & & \multicolumn{5}{|c|}{ Set operation computing models } & & & & \\
\hline & \multicolumn{12}{|c|}{$\begin{array}{l}\text { DNA/AuNP } \\
\text { DNA: B1 }\end{array}$} \\
\hline & \multicolumn{2}{|c|}{ (1) } & \multicolumn{2}{|c|}{ (2) } & \multicolumn{2}{|c|}{ (3) } & \multicolumn{2}{|c|}{ (4) } & \multicolumn{2}{|c|}{ (5) } & \multicolumn{2}{|c|}{ (6) } \\
\hline \multicolumn{13}{|l|}{ Input signals } \\
\hline DNA: A1 & 1 & 0 & 1 & 1 & 1 & 1 & 0 & 0 & 0 & 0 & 1 & 1 \\
\hline DNA: A3 & 1 & 1 & 1 & (2) & 0 & 0 & 1 & 1 & 0 & 0 & 1 & 1 \\
\hline DNA: A4 & 1 & 1 & 1 & 1 & 1 & (2) & 1 & 1 & 1 & 0 & 0 & 0 \\
\hline DNA: B2 & 1 & 1 & 1 & 1 & 1 & 1 & 1 & (0) & 1 & 1 & 1 & 0 \\
\hline \multirow{2}{*}{$\begin{array}{l}\text { Computing } \\
\text { results }\end{array}$} & 1 & 1 & 1 & 1 & 1 & 0 & 1 & 0 & 0 & 0 & 0 & 0 \\
\hline & H & y & y & U & $\psi$ & $y$ & 0 & $\nabla$ & 7 & $\nabla$ & $\theta$ & $y$ \\
\hline$x_{i}$ & \multicolumn{2}{|c|}{$\mathrm{X} 1=1$ or 0} & \multicolumn{2}{|c|}{$X 2=1$ or 0} & \multicolumn{2}{|c|}{$X 3=1$} & \multicolumn{2}{|c|}{$X 4=1$} & \multicolumn{2}{|c|}{ X5 no solution } & $\mathrm{X} 6 \mathrm{n}$ & solution \\
\hline
\end{tabular}

Figure 4 Set operation computing models results. The values in red circles represent input Xi.

(4) $\mathrm{A}=0, \mathrm{~B}=1, \mathrm{C}=1, \mathrm{D}=\mathrm{X}_{4}$;

(5) $\mathrm{A}=0, \mathrm{~B}=0, \mathrm{C}=\mathrm{X}_{5}, \mathrm{D}=1$;

(6) $\mathrm{A}=1, \mathrm{~B}=1, \mathrm{C}=0, \mathrm{D}=\mathrm{X}_{6}$.

Here take solving $X_{1}$ and $X_{4}$ for example, to show the general set computing process. To solve $X_{1}$, first, added given value DNA information signals A3, A4, B2. Then the additions of $\mathrm{X}_{1}$ (A1) were divided into two types: adding DNA strand $\mathrm{A} 1\left(\mathrm{X}_{1}=1\right)$ or not adding DNA strand $\mathrm{A} 1\left(\mathrm{X}_{1}=0\right)$ (Figure 4 (1) red circle). From the point of DNA/AuNP self-assembly, when DNA strand A3 was added, no matter input DNA strand A1 exists or not, the complete DNA self-assembly structures were constructed all the same, and AuNP were connected with each other (Figure 2). From the experimental phenomenon, the reaction system color changed from red to purple for AuNP aggregation. Base on computing model outputs, when $X_{1}=1$ or 0 , the results of set operation were 1 (Figure 4). Thus, the answers of $X_{1}$ are 1 or 0 . In the course of solving $X_{4}$, in the same way, added given value DNA information signals A1, A3, A4. Then, consider all possible answers of $\mathrm{X}_{4}$ (B2). Different from missing structure of DNA strand A1, the missing of DNA strand B2 would directly lead the whole self-assembly structure to dismiss, which served as arms to connected AuNP (Figure 2). Similar to experiment designs, only when $\mathrm{X}_{4}=1$, the reaction system color changed from red to purple. That means, when $X_{4}=1$, the value of set operation is 1 (Figure 4). In the same way, other solutions were able to be obtained: $X_{2}=1$ or $0 ; X_{3}=1 ; X_{5}$ has no solution; $X_{6}$ has no solution.

\subsection{The applications of molecular detecting}

Based on the logic computing models above, a simple method of molecular detection was developed using the color change of DNA/AuNP conjugates. By loading a virus gene sequence (H1N1 influenza, GenBank number: M76604) into the detecting nanodevice, the specific H1N1 gene could be detected. As shown in Figure 5, the detecting nanodevice changes color in response to a specific $\mathrm{H} 1 \mathrm{~N} 1$ gene sequence. By contrast, for control group DNA A1, there was no color change.

\section{Discussion}

In this paper, logic computing models were established using the technology of DNA/AuNP conjugates color change and DNA computing. Several abilities were demonstrated, including logic computing, set operation, and molecular detection. By adding specific information DNA strands, different DNA self-assembly structures are formed. With the change in DNA self-assembly structures, the color of the system changes from red to purple. In particular, several critical technologies are utilized in this molecular computing model: DNA self-assembly, DNA/AuNP conjugates and the color change by AuNP aggregation.

Compared with previous studies, this experiment is a little simple and still has some limitations. First, although the DNA strands used in this work are all designed and tested by software, some problems were also encountered, such as DNA strand mismatch and cross hybridization. However,

\begin{tabular}{l|c|c|c|}
\multicolumn{4}{c}{ H1N1 gene detecting model } \\
\hline $\begin{array}{l}\text { Model } \\
\text { component }\end{array}$ & control & \multicolumn{2}{|c|}{$\begin{array}{l}\text { DNA/AuNP } \\
\text { DNA: B3 }\end{array}$} \\
\hline Input signal & & & \\
H1N1 gene (A5) & 0 & 1 & 0 \\
DNA: A1 & 0 & 0 & 1 \\
\hline Results & 0 & 1 & 0 \\
& $y$ & $y$ & 3 \\
\hline
\end{tabular}

Figure 5 H1N1 gene molecular detection model. 
these problems had no influence on the computing results, which still demonstrated a strong dependence on well-designed DNA sequence. Second, the DNA self-assembly structures in this research are rather simple, and their computing ability is weak. Therefore, a future aim is to improve the complexity and ability of these logic computing models.

In this work, there are several major characteristics. First, in the computing process, the operations of DNA self-assembly are parallel large scale [28], and the accuracy and stability of computing are based on the high specific hybridization. Second, by the color change reaction, a tiny difference in molecules could be transformed into an obvious color change. The color change reaction is stable, and it is unnecessary to use large equipment (detected by naked eyes). Third, there is only one kind of DNA/AuNP conjugate used in this experiment. This not only saved the time and cost, but also reduced the complexity of DNA sequence design. Lastly, combined with molecular detection and diagnostics, there will be many potential applications of DNA/AuNP logic models.

Using DNA/AuNP conjugates, nanoparticles enlarge the abilities of DNA as an organic compound, and provide free moving electrons. On the other hand, DNA provides the chemically decorated sites and specific hybridization sites. By the precise conjugation of DNA and nanoparticles, the whole nanosystem is reconstructed step by step. In addition, because the nanoparticle is of quantum size and has a nanosurface, molecular computing and quantum computing will develop together, and provide more possibilities for future computing technology. With the development of molecular operations, DNA computing will have further applications in information processing, cryptography, intelligent nanodevices, and nanoelectronics.

The authors sincerely acknowledge all the referee's suggestions for this paper. This work was supported by the National Natural Science Foundation of China (60910002, 61033003, 60974112, 60971085, and 30970969), National High-Tech Research and Development Program of China (2009AA01Z413), Ph.D. Programs Foundation of the Ministry of Education of China (20070001020), and the Postdoctoral Science Foundation of China (20100480128).

1 Lipton R J. DNA solution of hard computational problems. Science, 1995, 268: 542-545

2 Adleman L M. Molecular computation of solutions to combinatorial problems. Science, 1994, 266: 1021-1024

3 Ouyang Q, Kaplan P D, Liu S, et al. DNA solution of the maximal clique problem. Science, 1997, 278: 446-449

4 Liu Q H, Wang L M, Frutos A G, et al. DNA computing on surfaces. Nature, 2000, 403: 175-178

5 Kodama T, Jain A, Goodson K E. Heat conduction through a
DNA-gold composite. Nano Lett, 2009, 9: 2005-2009

6 Chen X, Wang Y, Liu Q, et al. Construction of molecular logic gates with a DNA-cleaving deoxyribozyme. Angew Chem Int Ed, 2006, 45: 1759-1762

7 Niazov T, Baron R, Katz E, et al. Concatenated logic gates using four coupled biocatalysts operating in series. Proc Natl Acad Sci USA, 2006, 103: 17160-17163

8 Privman M, Tam T K, Pita M, et al. Switchable electrode controlled by enzyme logic network system: Approaching physiologically regulated bioelectronics. J Am Chem Soc, 2009, 131: 1314-1321

9 Mu L, Shi W, She G, et al. Fluorescent logic gates chemically attached to silicon nanowires. Angew Chem Int Ed, 2009, 48: 1-5

10 Amir L, Tam T K, Pita M, et al. Biofuel cell controlled by enzyme logic systems. J Am Chem Soc, 2009, 131: 826-832

11 Szaciowski K, Macyk W, Stochel G. Light-driven OR and XOR programmable chemical logic gates. J Am Chem Soc, 2006, 128: 4550-4551

12 Yurke B, Turberfield A J, Allen P M Jr, et al. A DNA-fuelled molecular machine made of DNA. Nature, 2000, 406: 605-608

13 Turberfield A J, Mitchell J C. DNA fuel for free-running nanomachines. Phys Rev Lett, 2003, 90: 118802

14 Shin J-S, Pierce N A. Rewritable memory by controllable nanopatterning of DNA. Nano Lett, 2004, 4: 905-909

15 Seelig G, Yurke B, Winfree E. Catalyzed relaxation of a metastable DNA fuel. J Am Chem Soc, 2006, 128: 12211-12220

16 Aldaye F A, Sleiman H F. Sequential self-assembly of a DNA hexagon as a template for the organization of gold nanoparticles. Angew Chem Int Ed, 2006, 118: 2262-2267

17 Aldaye F A, Sleiman H F. Dynamic DNA templates for discrete gold nanoparticle assemblies control of geometry, modularity, write erase and structural switching. J Am Chem Soc, 2007, 129: 4130-4131

18 Sharma J, Chhabra R, Cheng A, et al. Control of self-assembly of DNA tubules through integration of gold nanoparticles. Science, 2009, 323: 112-116

19 Mastroianni A J, Claridge S A, Alivisatos A P. Pyramidal and chiral groupings of gold nanocrystals assembled using DNA scaffolds. J Am Chem Soc, 2009, 131: 8455-8459

20 Mao C D, LaBean T H, Reif J H, et al. Logical computation using algorithmic self-assembly of DNA triplecrossover molecules. Nature, 2000, 407: 493-496

21 Millian N S, Darko S. A deoxyribozyme-based molecular automaton. Nat Biotechnol, 2003, 21: 1069-1074

22 Seelig G, Soloveichik D, Zhang Y D, et al. Enzyme-free nucleic acid logic circuits. Science, 2006, 314: 1585-1588

23 Elbaz J, Lioubashevski O, Wang F, et al. DNA computing circuits using libraries of DNAzyme subunits. Nat Biotechnol, 2010, 5: $417-422$

$24 \mathrm{Gu} \mathrm{H}$, Chao J, Xiao S, et al. Dynamic patterning programmed by DNA tiles captured on a DNA origami substrate. Nat Nanotechnol, 2009, 4: 245-248

25 Goodman R P, Schaap I A T, Tardin C F, et al. Rapid chiral assembly of rigid DNA building blocks for molecular nanofabrication. Science, 2005, 310: 1661-1665

26 Storhoff J J, Lucas A D, Garimella V, et al. Homogeneous detection of unamplified genomic DNA sequences based on colorimetric scatter of gold nanoparticle probes. Nat Biotechnol, 2004, 22: 883-887

27 Mirkin C A, Letsinger R L, Mucic R C, et al. A DNA based method for rationally assembling nanoparticles into macroscopic materials. Nature, 1996, 382: 607-609

28 Majid D. A new solution for maximal clique problem based sticker model. Biosystems, 2009, 95: 145-149

Open Access This article is distributed under the terms of the Creative Commons Attribution License which permits any use, distribution, and reproduction in any medium, provided the original author(s) and source are credited. 